\title{
Applications of order-theoretic fixed point theorems to discontinuous quasi-equilibrium problems
}

\author{
Congjun Zhang ${ }^{1}$ and Yuehu Wang ${ }^{2^{*}}$
}

"Correspondence:
wyhmath@163.com
${ }^{2}$ School of Mathematical Sciences,
Anhui University, Hefei, 230601,
P.R. China
Full list of author information is
available at the end of the article

available at the end of the article

\begin{abstract}
In this paper, we apply order-theoretic fixed point theorems and isotone selection theorems to study quasi-equilibrium problems. Some existence theorems of solutions to quasi-equilibrium problems are obtained on Hilbert lattices, chain-complete lattices and chain-complete posets, respectively. In contrast to many papers on equilibrium problems, our approach is order-theoretic and all results obtained in this paper do not involve any topological continuity with respect to the considered mappings.
\end{abstract}

Keywords: order-theoretic fixed points; quasi-equilibrium problems; Hilbert lattices; posets; discontinuous; existence

\section{Introduction}

Let $X$ be a given set and $C$ be a subset of $X$. Let $T: C \rightarrow 2^{C} \backslash\{\emptyset\}$ be a set-valued mapping. Let $f: C \times C \rightarrow R$ be a given function. The quasi-equilibrium problem (shortly QEP) is to find $x^{*} \in T\left(x^{*}\right)$ such that

$$
f\left(x^{*}, y\right) \geq 0 \quad \text { for all } y \in T\left(x^{*}\right)
$$

This problem was introduced by Noor and Oettli in [1]. In particular, if $T(x)=C$ for any $x \in$ $C$, then the quasi-equilibrium problem is reduced to the following classical equilibrium problem (shortly EP), which is to find $x^{*} \in C$ such that

$$
f\left(x^{*}, y\right) \geq 0 \text { for all } y \in C \text {. }
$$

EP was initially introduced by Blum and Oettli [2]. It is well known that EP include variational inequality problems, fixed point problems, complementarity problems, saddle point problems and Nash equilibrium problems as special cases (see, e.g., [1-4]).

If there is at least one solution to QEP (EP), then we say QEP (EP) is solvable. For studying the existence of solutions to QEP and EP, various methods have been developed, for instance, KKM theorem, F-KKM theorem, Ekeland's variational principles, topological fixed point theorems, auxiliary principle and many others (see, e.g., [5-18]). Among these methods, a variety of continuity or Brezis-type monotonicity conditions of $f$ are

(c) 2015 Zhang and Wang; licensee Springer. This article is distributed under the terms of the Creative Commons Attribution 4.0 International License (http://creativecommons.org/licenses/by/4.0/), which permits unrestricted use, distribution, and reproduction in any medium, provided you give appropriate credit to the original author(s) and the source, provide a link to the Creative Commons license, and indicate if changes were made. 
commonly necessary. For instance, Cubiotti [10] studies the lower semicontinuous quasiequilibrium problems in topological vector spaces. Al-Homidan and Ansari [15] consider the systems of generalized vector quasi-equilibrium problems on topological semilattice spaces and establish some existence results for solutions of systems of generalized vector quasi-equilibrium problems and their special cases, where they require the considered mappings to be upper semicontinuous or lower semicontinuous. However, this approach may fail when the topological continuity of $f$ is unknown. On the other hand, we note that Fujimoto [19], Chitra and Subrahmanyam [20] and Borwein and Dempster [21] have adopted an order-theoretic approach for studying the nonlinear complementarity problems, where the mappings only need to satisfy some order-preserving properties. Along this line, Nishimura and Ok [22] have extended these results to the case of (generalized) variational inequalities on Hilbert lattices. Very recently, Li and Ok [23] used the variational characterization and order-preservation properties of a generalized metric projection operator to study the (generalized) variational inequalities on Banach lattices.

Motivated and inspired by the work of Li and Ok [23], Cubiotti [10], Al-Homidan and Ansari [15] et al., in this paper we aim to study the quasi-equilibrium problems by using order-theoretic methods, where we do not require $f$ to be continuous and semicontinuous. Furthermore, it is worthy to mention that some of order-theoretic methods and techniques developed in variational inequality problems are not suitable for studying quasiequilibrium problems. Actually, the existence results for variational inequalities are based on the fact that the solutions to variational inequalities coincide with the fixed points of the self-correspondence $\pi_{C} \circ\left(\operatorname{id}_{X}-\Gamma\right)$, where $\mathrm{id}_{X}$ is the identity mapping on $X$ and $\Gamma$ is the involved mapping and $\pi_{C}$ is the metric projection operator onto $C$. To guarantee that $\pi_{C} \circ\left(\operatorname{id}_{X}-\Gamma\right)$ has fixed points, we always need the metric projection operator to be order-preserving. That is, the (generalized) metric projection operators play crucial roles in dealing with the variational inequalities. Unfortunately, there is no (generalized) metric projection operator in quasi-equilibrium problems. Therefore, it is necessary to provide some other techniques to circumvent this difficulty. In this paper, we use the ordertheoretic fixed point theorem and isotone selection theorems to obtain some existence theorems for discontinuous quasi-equilibrium problems.

The rest of the present paper can be summarized as follows. Section 2 is devoted to some basic concepts on posets as well as the order-preserving properties of correspondences. In Section 3, based on the order-theoretic fixed point theorem of Nishimura and Ok, we study the existence of solutions for quasi-equilibrium problems and equilibrium problems on Hilbert lattices. In Section 4, we apply the isotone selection theorems and the order-theoretic fixed point theorem introduced by Tarski to establish some existence results for quasi-equilibrium problems and equilibrium problems on chain-complete lattices, which are equipped with neither an algebraic structure nor a topological structure. Furthermore, we also apply the order-theoretic fixed point theorems introduced by Li to establish some existence results for quasi-equilibrium problems and equilibrium problems on chain-complete posets.

\section{Preliminaries}

In this section, we recall some basic concepts about Hilbert lattices as well as several useful lemmas. For more details, the readers are referred to [24-26]. 


\subsection{Some concepts in poset}

A poset is an ordered pair $(X, \succcurlyeq)$, where $X$ is a nonempty set and $\succcurlyeq$ denotes the partial order defined on $X$. For each $x \in(X, \succcurlyeq)$, we define $x^{\uparrow}=\{y \in(X, \succcurlyeq): y \succcurlyeq x\}$ and $x_{\downarrow}=\{y \in$ $(X, \succcurlyeq): x \succcurlyeq y\}$. In turn, for any nonempty subset $S$, we define $S^{\uparrow}=\bigcup\left\{x^{\uparrow}: x \in S\right\}$ and $S^{\downarrow}=$ $\bigcup\left\{x^{\downarrow}: x \in S\right\}$. We say that an element $x$ of $(X, \succcurlyeq)$ is an $\succcurlyeq$-upper bound for $S$ if $x \succcurlyeq S$, that is, $x \succcurlyeq y$ for each $y \in S$. The notation $S \succcurlyeq x$ is similarly understood. We say that $S$ is $\succcurlyeq$-bounded from above if $x \succcurlyeq S$ for some $x \in(X, \succcurlyeq)$ and $\succcurlyeq$-bounded from below if $S \succcurlyeq x$ for some $x \in(X, \succcurlyeq)$. In turn, $S$ is said to be $\succcurlyeq$-bounded if it is $\succcurlyeq$-bounded from above and below. Particularly, if $x \in S$ and $x$ is an $\succcurlyeq$-upper bound for $S$, then we say that $x$ is the $\succcurlyeq$-maximum in $S$. The $\succcurlyeq$-minimum element of $S$ is similarly defined. We say that $x$ is the $\succcurlyeq$-maximal element of $S$ if $x \in S$ and $y \succcurlyeq x$ does not hold for any $y \in S \backslash\{x\}$. Similarly, $x$ is said to be the $\succcurlyeq$-minimal element of $S$ if $x \in S$ and $x \succcurlyeq y$ does not hold for any $y \in S \backslash\{x\}$. A nonempty subset $S$ of $X$ is said to be a $\succcurlyeq$-chain in $X$ if either $x \succcurlyeq y$ or $y \succcurlyeq x$ hold for each $x, y \in S$.

The $\succcurlyeq$-supremum of $S$ is the $\succcurlyeq$-minimum of the set of all $\succcurlyeq$-upper bounds for $S$, and is denoted by $\bigvee_{X} S$. The $\succcurlyeq$-infimum of $S$ which is denoted by $\bigwedge_{X} S$ is defined similarly. As is conventional, we denote $\bigvee_{X}\{x, y\}$ as $x \vee y$ and $\bigwedge_{X}\{x, y\}$ as $x \wedge y$ for any $x, y$ in $(X, \succcurlyeq)$. If $x \vee y$ and $x \wedge y$ exist for every $x$ and $y$ in $(X, \succcurlyeq)$, then we say that $(X, \succcurlyeq)$ is a lattice, and if $\bigvee_{X} S$ and $\bigwedge_{X} S$ exist for every nonempty $\succcurlyeq$-bounded $S \subseteq(X, \succcurlyeq)$, then we say that $(X, \succcurlyeq)$ is a Dedekind complete lattice. If $Y$ is a nonempty subset of $(X, \succcurlyeq)$ which contains $\bigvee_{X}\{x, y\}$ and $\bigwedge_{X}\{x, y\}$ for every $x, y \in Y$, then it is said to be a $\succcurlyeq$-sublattice of $(X, \succcurlyeq)$. In turn, if $Y$ contains $\bigvee_{X} S$ and $\bigwedge_{X} S$ for every nonempty $S \subseteq Y$, then $Y$ is said to be a complete $\succcurlyeq$-sublattice of $(X, \succcurlyeq)$.

Let $A$ be a nonempty subset of $X, A$ is said to be inductive if every chain in $A$ has an upper bound in $A$. Moreover, $A$ is said to be chain-complete if every chain $C$ in $A$ possesses its supremum in $A$.

\subsection{Order-preservation for correspondences}

For any lattices $\left(X, \succcurlyeq_{X}\right)$ and $\left(Y, \succcurlyeq_{Y}\right)$, we say that a map $F: X \rightarrow Y$ is order-preserving if $x \succcurlyeq_{X} y$ implies $F(x) \succcurlyeq_{Y} F(y)$ for every $x, y \in X$. In turn, if $\Gamma: X \rightarrow 2^{Y}$ is a set-valued mapping, we say that $\Gamma$ is upper order-preserving if $x \succcurlyeq_{X} y$ implies that $\Gamma(y)=\emptyset$, or for every $y^{\prime} \in \Gamma(y)$ there is $x^{\prime} \in \Gamma(x)$ such that $x^{\prime} \succcurlyeq_{Y} y^{\prime}$. Upper order-reversing maps are defined dually. Similarly, $\Gamma$ is lower order-preserving if $x \succcurlyeq_{X} y$ implies that $\Gamma(x)=\emptyset$, or for every $x^{\prime} \in \Gamma(x)$ there is $y^{\prime} \in \Gamma(y)$ such that $x^{\prime} \succcurlyeq_{Y} y^{\prime}$. $\Gamma$ is order-preserving if it is both upper and lower order-preserving. If $\left(X, \succcurlyeq_{X}\right)$ and $\left(Y, \succcurlyeq_{Y}\right)$ are subposets of a given poset $(Z, \succcurlyeq)$, then we use the phrase $\succcurlyeq$-preserving instead of order-preserving. $\Gamma$ is said to be upper $\succcurlyeq$-bound if there exists $y^{*} \in Y$ such that $\bigvee_{Y} \Gamma(x)$ exists and

$$
y^{*} \succcurlyeq \bigvee_{Y} \Gamma(x)
$$

$\Gamma$ is said to have upper bound $\succcurlyeq$-closed values if $x \in X$ implies $\Gamma(x)=\emptyset$ or

$$
\bigvee_{Y} \Gamma(x) \in \Gamma(x)
$$

$\Gamma$ is said to be lower $\succcurlyeq$-bound if there exists $y_{*} \in Y$ such that $\bigwedge_{Y} \Gamma(x)$ exists and

$$
\bigwedge_{Y} \Gamma(x) \succcurlyeq y_{*} .
$$


$\Gamma$ is said to have lower bound $\succcurlyeq$-closed values if $x \in X$ implies $\Gamma(x)=\emptyset$ or

$$
\bigwedge_{Y} \Gamma(x) \in \Gamma(x)
$$

Definition 2.1 Let $\Gamma: X \rightarrow 2^{Y} \backslash\{\emptyset\}$ be a set-valued mapping. A selection for $\Gamma$ is a singlevalued function $F: X \rightarrow Y$ such that $F(x) \in \Gamma(x)$ for each $x \in X$. An isotone selection is a selection which is order-preserving.

Lemma 2.1 (see [27]) Let $(X, \succcurlyeq)$ be a poset, and let $\Gamma: X \rightarrow 2^{X} \backslash\{\emptyset\}$ be a set-valued mapping. If $\Gamma$ is upper $\succcurlyeq$-preserving and has upper bound $\succcurlyeq$-closed values, then the singlevalued mapping $F: X \rightarrow X$ defined by $F(x)=\bigvee_{X} \Gamma(x)$ is an isotone selection for $\Gamma$.

Lemma 2.2 (see [27]) Let $(X, \succcurlyeq)$ be a poset, and let $\Gamma: X \rightarrow 2^{X} \backslash\{\emptyset\}$ be a set-valued mapping. If $\Gamma$ is lower $\succcurlyeq$-preserving and has lower bound $\succcurlyeq$-closed values, then the single-valued mapping $F: X \rightarrow X$ defined by $F(x)=\bigwedge_{X} \Gamma(x)$ is an isotone selection for $\Gamma$.

\subsection{Hilbert lattice and several notations}

We say that $(X, \succcurlyeq)$ is a Hilbert lattice if $X$ is a Hilbert space with the inner product $\langle\cdot, \cdot\rangle$ and with the induced norm $\|\cdot\|$ and $X$ is also a poset with the partial order $\succcurlyeq$ satisfying the following conditions:

(i) $(X, \succcurlyeq)$ is a lattice.

(ii) The mapping $\alpha \operatorname{id}_{X}+z$ is a $\succcurlyeq$-preserving self-mapping on $X$ for every $z \in X$ and positive number $\alpha$, where $\operatorname{id}_{X}$ denotes the identical mapping on $X$.

(iii) The norm $\|\cdot\|$ on $X$ is compatible with the partial order $\succcurlyeq$, that is, $|x| \succcurlyeq|y|$ implies $\|x\| \geq\|y\|$, where $|z|=(z \vee \mathbf{0})+(-z \vee \mathbf{0})$ for every $z \in X$ and $\mathbf{0}$ denotes the origin of $X$.

At the end of this section, we introduce a notation, which will be used frequently in the following sections. Let $X$ be a given set and let $C$ be a subset of $X$. Let $T: C \rightarrow 2^{C} \backslash\{\emptyset\}$ be a set-valued mapping. Throughout the paper, the set $\{x \in C: x \in T(x)\}$ is always denoted by $E$.

\section{Solvability of quasi-equilibrium problems on Hilbert lattices}

For studying generalized variational inequalities, Nishimura and Ok introduced the following order-theoretic fixed point theorem on Hilbert lattices.

Lemma 3.1 (see [22]) Let $(X, \succcurlyeq)$ be a separable Hilbert lattice and $C$ be a weakly compact and convex $\succcurlyeq$-sublattice of $X$. Then every upper $\succcurlyeq$-preserving and compact-valued correspondence $F: C \rightarrow 2^{C} \backslash\{\emptyset\}$ has a fixed point.

Based on Lemma 3.1, we can get an existence theorem for quasi-equilibrium problems as follows.

Theorem 3.1 Let $(X, \succcurlyeq)$ be a separable Hilbert lattice, and let $C$ be a weakly compact convex $\succcurlyeq$-sublattice of $X$. Let $f: C \times C \rightarrow R$ be a bifunction and $T: C \rightarrow 2^{C} \backslash\{\emptyset\}$ be a set-valued mapping. Assume that the following conditions hold:

(i) $f(x, x) \geq 0$ for any $(x, x) \in C \times C$. 
(ii) $T$ is upper $\succcurlyeq$-preserving and compact-valued. $x \nsucc C \backslash E$ for any $x \in E$.

(iii) The set-valued mapping $\Phi: C \rightarrow 2^{C}$ defined by setting

$$
\Phi(x)=\{y \in T(x): f(x, y)<0\}
$$

is upper $\succcurlyeq$-preserving and compact-valued.

Then QEP (1.1) is solvable.

Proof We claim that there exists $x^{*} \in E$ such that $\Phi\left(x^{*}\right)=\emptyset$. Arguing by contradiction, assume $\Phi(x) \neq \emptyset$ for all $x \in E$, then we can define a set-valued mapping $\Psi: C \rightarrow 2^{C} \backslash\{\emptyset\}$ by

$$
\Psi(x)= \begin{cases}\Phi(x) & \text { if } x \in E, \\ T(x) & \text { if } x \in C \backslash E .\end{cases}
$$

Next, we divide the rest of the proof into two steps.

Step 1 . Show that $\Psi$ is upper $\succcurlyeq$-preserving.

Take any $x_{1}, x_{2} \in C$ with $x_{1} \succcurlyeq x_{2}$ and pick an arbitrary $y_{2} \in \Psi\left(x_{2}\right)$. We wish to find $y_{1} \in$ $\Psi\left(x_{1}\right)$ such that $y_{1} \succcurlyeq y_{2}$. To this end, we consider the following three cases.

Case I. If $x_{1}, x_{2} \in E$, then the upper $\succcurlyeq$-preservation of $\Psi$ is equivalent to the upper $\succcurlyeq$-preservation of $\Phi$. Thus, we only need to prove that $\Phi$ is upper $\succcurlyeq$-preserving. From assumption (iii), it is obvious.

Case II. If $x_{1}, x_{2} \in C \backslash E$, then the upper $\succcurlyeq$-preservation of $\Psi$ is reduced to the upper $\succcurlyeq$-preservation of $T$. It follows immediately from assumption (ii).

Case III. If $x_{1} \in C \backslash E$ and $x_{2} \in E$, then $y_{2} \in \Psi\left(x_{2}\right)$ is reduced to $y_{2} \in \Phi\left(x_{2}\right)$, which implies that $y_{2} \in T\left(x_{2}\right)$. Again, since $T$ is upper $\succcurlyeq$-preserving, there exists $y_{1} \in T\left(x_{1}\right)=\Psi\left(x_{1}\right)$ such that $y_{1} \succcurlyeq y_{2}$.

Furthermore, since $x \nsucc C \backslash E$ for any $x \in E, x_{1} \succcurlyeq x_{2}$ does not hold for any $x_{1} \in E$ and $x_{2} \in C \backslash E$. Above all, from Case I, Case II and Case III, we conclude that $\Psi$ is upper $\succcurlyeq$-preserving on $C$.

Step 2. Prove that $\Psi$ has a fixed point.

Since $C$ is a weakly compact and convex $\succcurlyeq$-sublattice of $X$ and $\Psi$ is upper $\succcurlyeq$-preserving and compact-valued, $\Psi$ has a fixed point by Lemma 3.1. Denote this fixed point by $\bar{x}$. Noting that $\{x \in C: x \in \Psi(x)\} \subseteq E$, we get $\bar{x} \in E \cap \Phi(\bar{x})$, and hence we have $f(\bar{x}, \bar{x})<0$, which contradicts with (i). Therefore, there exists $x^{*} \in E$ such that $\Phi\left(x^{*}\right)=\emptyset$. That is, $x^{*} \in T\left(x^{*}\right)$ and $f\left(x^{*}, y\right) \geq 0$ for all $y \in T\left(x^{*}\right)$.

Example 3.1 It is easy to construct examples of a set-valued mapping $T$ that satisfies the assumption (ii) of Theorem 3.1. For instance, take $X=(R, \geq)$ and $C=[1,3]$. Define $T:[1,3] \rightarrow 2^{[1,3]}$ by setting

$$
T(x)= \begin{cases}{[1,2]} & \text { if } x \in[1,2], \\ \{2\} & \text { if } x \in(2,3] .\end{cases}
$$

It is easy to check that $E=[1,2]$ and $C \backslash E=(2,3]$, and obviously $x \leq C \backslash E$ for every $x \in E$. 
In Theorem 3.1, the new mapping $\Phi$ related to the considered mapping $f$ is involved in assumption (iii). It is a kind of burdensome for the applications of Theorem 3.1. Hence, to whittle down the nuisance caused by $\Phi$, it is desirable to find some different conditions only on $f$ such that $\Phi$ is still upper $\succcurlyeq$-preserving. Therefore, the following result is obtained.

Theorem 3.2 Let $(X, \succcurlyeq)$ be a separable Hilbert lattice, and let $C$ be a weakly compact convex $\succcurlyeq$-sublattice of $X$. Let $f: C \times C \rightarrow R$ be a bifunction and $T: C \rightarrow 2^{C} \backslash\{\emptyset\}$ be a set-valued mapping. Assume that the following conditions hold:

(i) $f(x, x) \geq 0$ for any $(x, x) \in C \times C$.

(ii) $T$ is upper $\succcurlyeq$-preserving and compact-valued. $x \nsucc C \backslash E$ for any $x \in E$.

(iii) $f(\cdot, y)$ is order-reversing for any $y \in C$ and $f(x, \cdot)$ is order-reversing for any $x \in C$.

Moreover, the set $\{y \in C: f(x, y)<0\}$ is closed for any $x \in C$.

Then QEP (1.1) is solvable.

Proof Define a set-valued mapping $\Phi: C \rightarrow 2^{C}$ by setting

$$
\Phi(x)=\{y \in T(x): f(x, y)<0\}
$$

For applying Theorem 3.1, we only need to prove that $\Phi$ is upper $\succcurlyeq$-preserving and compact-valued. In fact, take any $x_{1}, x_{2} \in C$ with $x_{1} \succcurlyeq x_{2}$ and pick an arbitrary $y_{2} \in \Phi\left(x_{2}\right)$. We wish to find $y_{1} \in \Phi\left(x_{1}\right)$ such that $y_{1} \succcurlyeq y_{2}$.

Since $y_{2} \in \Phi\left(x_{2}\right)$, we have

$$
y_{2} \in T\left(x_{2}\right) \text { and } f\left(x_{2}, y_{2}\right)<0 \text {. }
$$

Since $T$ is upper $\succcurlyeq$-preserving by assumption (ii), there exists $y_{1} \in T\left(x_{1}\right)$ such that $y_{1} \succcurlyeq y_{2}$. Noting that $x_{1} \succcurlyeq x_{2}$ and that $f\left(\cdot, y_{1}\right)$ is order-reversing by assumption (iii), we have

$$
f\left(x_{2}, y_{1}\right) \geq f\left(x_{1}, y_{1}\right)
$$

Again, since $y_{1} \succcurlyeq y_{2}$ and $f\left(x_{2}, \cdot\right)$ is order-reversing by assumption (iii), we get

$$
f\left(x_{2}, y_{2}\right) \geq f\left(x_{2}, y_{1}\right)
$$

Combining (3.2), (3.3) and (3.4), we obtain

$$
f\left(x_{1}, y_{1}\right)<0
$$

Hence, $\Phi$ is upper $\succcurlyeq$-preserving on $C$.

On the other hand, since $\{y \in C: f(x, y)<0\}$ is closed for any $x \in C$ by assumption (iii) and $T(x)$ is a compact subset of $C$ for any $x \in C$ by assumption (ii), we conclude that $\Phi$ is compact-valued. Therefore, $\Phi$ satisfies the assumption (iii) of Theorem 3.1, and then quasi-equilibrium problem (1.1) has a solution.

In particular, if $T(x)=C$ for any $x \in C$, then we can deduce the following results from Theorem 3.1 and Theorem 3.2. 
Corollary 3.1 Let $(X, \succcurlyeq)$ be a separable Hilbert lattice, and let $C$ be a compact convex $\succcurlyeq$-sublattice of $X$. Let $f: C \times C \rightarrow R$ be a bifunction. Assume that the following conditions hold:

(i) $f(x, x) \geq 0$ for any $(x, x) \in C \times C$.

(ii) The set-valued mapping $\Phi: C \rightarrow 2^{C}$ defined by setting

$$
\Phi(x)=\{y \in C: f(x, y)<0\}
$$

is upper $\succcurlyeq$-preserving and compact-valued.

Then EP (1.2) is solvable.

Corollary 3.2 Let $(X, \succcurlyeq)$ be a separable Hilbert lattice, and let $C$ be a compact convex $\succcurlyeq$-sublattice of $X$. Let $f: C \times C \rightarrow R$ be a bifunction. Assume that the following conditions hold:

(i) $f(x, x) \geq 0$ for any $(x, x) \in C \times C$.

(ii) $f(\cdot, y)$ is order-reversing for any $y \in C$ and $f(x, \cdot)$ is order-reversing for any $x \in C$.

Moreover, the set $\{y \in C: f(x, y)<0\}$ is compact for any $x \in C$.

Then EP (1.2) is solvable.

In fact, the assumption (ii) of Corollary 3.2 can be weakened as follows.

Corollary 3.3 Let $(X, \succcurlyeq)$ be a separable Hilbert lattice, and let $C$ be a compact convex $\succcurlyeq$-sublattice of $X$. Let $f: C \times C \rightarrow R$ be a bifunction. Assume that the following conditions hold:

(i) $f(x, x) \geq 0$ for any $(x, x) \in C \times C$.

(ii)' $f(\cdot, y)$ is order-reversing for any $y \in C$, and the set $\{y \in C: f(x, y)<0\}$ is compact for any $x \in C$.

Then EP (1.2) is solvable.

Proof Define a set-valued mapping $\Phi: C \rightarrow 2^{C}$ by setting

$$
\Phi(x)=\{y \in C: f(x, y)<0\} .
$$

For applying Theorem 3.1, we only need to prove that $\Phi$ is upper $\succcurlyeq$-preserving. In fact, take any $x_{1}, x_{2} \in C$ with $x_{1} \succcurlyeq x_{2}$ and pick an arbitrary $y_{2} \in \Phi\left(x_{2}\right)$. Since $y_{2} \in \Phi\left(x_{2}\right)$, we have $y_{2} \in C$ and $f\left(x_{2}, y_{2}\right)<0$. Again, since $f\left(\cdot, y_{2}\right)$ is order-reversing, we get $f\left(x_{2}, y_{2}\right) \geq f\left(x_{1}, y_{2}\right)$. Choose $y_{1}=y_{2}$, then $y_{1} \in C$ and $f\left(x_{1}, y_{1}\right)<0$, which implies that $y_{1} \in \Phi\left(x_{1}\right)$. Hence, $\Phi$ is upper $\succcurlyeq$-preserving. By Theorem 3.1, EP (1.2) is solvable.

Remark 3.1 Actually, based on the dual version of Zorn's lemma and Lemma 3.1, a fixed point theorem for lower $\succcurlyeq$-preserving correspondence can be obtained. Applying this new fixed point theorem, we can explore some existence theorems for quasi-equilibrium problems and equilibrium problems under the condition of lower $\succcurlyeq$-preservation.

Remark 3.2 In order to guarantee that condition (iii) in Theorems 3.1 and 3.2 holds, the continuity is not necessary. Indeed, we can give an example as follows. Take Theorem 3.1 
for example. Let $(X, \succcurlyeq)=(R, \geq)$ and $C=[0,2] \subseteq R$. Denote by $D$ the set $\{(x, y) \in[0,2] \times$ $[0,2]: x-y \geq 1\}$. Define a mapping $f:[0,2] \times[0,2] \rightarrow R$ by

$$
z=f(x, y)= \begin{cases}\frac{2}{3} y-\frac{2}{3} x+\frac{2}{3}, & (x, y) \in[0,2] \times[0,2] \backslash D, \\ y-x, & (x, y) \in D\end{cases}
$$

and define a set-valued mapping $T: C \rightarrow 2^{C} \backslash\{\emptyset\}$ by

$$
T(x)= \begin{cases}{\left[0, \frac{1}{2}\right],} & x \in\left[0, \frac{1}{2}\right] \\ \left\{\frac{1}{2}\right\}, & x \in\left(\frac{1}{2}, 2\right] .\end{cases}
$$

We can check that $f$ and $T$ satisfy all the conditions (including assumption (iii)) in Theorem 3.1, but $f$ is discontinuous. Furthermore, if we take $\hat{x}=\frac{1}{2}$, then we have

$$
f\left(\frac{1}{2}, y\right) \geq 0 \quad \text { for all } y \in T\left(\frac{1}{2}\right)
$$

which implies $\hat{x}=\frac{1}{2}$ is a solution to a quasi-equilibrium problem. Similarly, examples for Theorem 3.2 can also be given.

\section{Solvability of quasi-equilibrium problems on chain-complete lattices and chain-complete posets}

In this section, we explore several existence theorems on chain-complete lattices and chain-complete posets, on which there is neither a topological structure nor an algebraic structure.

Firstly, let us recall the following order-theoretic fixed point theorem, which was introduced by Tarski [28] in 1955.

Lemma 4.1 (see [28]) Let $(X, \succcurlyeq)$ be a chain-complete lattice, and let $F: X \rightarrow X$ be an order-preserving single-valued mapping. If there is $\hat{x} \in X$ with $F(\hat{x}) \succcurlyeq \hat{x}$, then $F$ has a fixed point.

Theorem 4.1 Let $(X, \succcurlyeq)$ be a poset and let $C$ be a chain-complete $\succcurlyeq$-sublattice of $X$. Let $f: C \times C \rightarrow R$ be a bifunction and $T: C \rightarrow 2^{C} \backslash\{\emptyset\}$ be a set-valued mapping. Assume that the following conditions hold:

(i) $f(x, x) \geq 0$ for any $(x, x) \in C \times C$.

(ii) $T$ is upper $\succcurlyeq$-preserving and has upper bound $\succcurlyeq$-closed values. $x \nsucc C \backslash E$ for any $x \in E$.

(iii) The set-valued mapping $\Phi: C \rightarrow 2^{C}$ defined by setting

$$
\Phi(x)=\{y \in T(x): f(x, y)<0\}
$$

is upper $\succcurlyeq$-preserving and has upper bound $\succcurlyeq$-closed values.

(iv) There is $\hat{x} \in C \backslash E$ such that $\bigvee_{C} T(\hat{x}) \succcurlyeq \hat{x}$, or there is $\hat{x} \in E$ such that $\bigvee_{C} \Phi(\hat{x}) \succcurlyeq \hat{x}$. Then QEP (1.1) is solvable. 
Proof We claim that there exists $x^{*} \in E$ such that $\Phi\left(x^{*}\right)=\emptyset$. Arguing by contradiction, assume $\Phi(x) \neq \emptyset$ for all $x \in E$. By the same argument as that in Theorem 3.1, we can define the set-valued mapping $\Psi: C \rightarrow 2^{C} \backslash\{\emptyset\}$ and prove that $\Psi$ is upper $\succcurlyeq$-preserving. From assumption (ii) and assumption (iii), it follows that $\Psi$ is upper bound $\succcurlyeq$-closed. From Lemma 2.1, there is an isotone selection $\psi$ for $\Psi$. From assumption (iv), there exists $\hat{x} \in C$ such that $\psi(\hat{x}) \succcurlyeq \hat{x}$. Since $C$ is a chain-complete $\succcurlyeq$-sublattice of $X$, therefore, by Lemma 4.1, there exists $\bar{x}$ in $C$ such that $\bar{x}=\psi(\bar{x}) \in \Psi(\bar{x})$. Since $\{x \in C: x \in \Psi(x)\} \subseteq E$, we get $\bar{x} \in E \cap$ $\Phi(\bar{x})$. In particular, we have $f(\bar{x}, \bar{x})<0$, which contradicts with assumption (i). Therefore, there exists $x^{*} \in E$ such that $\Psi\left(x^{*}\right)=\emptyset$. That is, $x^{*} \in T\left(x^{*}\right)$ and $f\left(x^{*}, y\right) \geq 0$ for all $y \in$ $T\left(x^{*}\right)$.

Replacing the assumption (iii) of Theorem 4.1 by some conditions on $f$, we can obtain the following results.

Theorem 4.2 Let $(X, \succcurlyeq)$ be a poset and let $C$ be a chain-complete $\succcurlyeq$-sublattice of $X$. Let $f: C \times C \rightarrow R$ be a bifunction and $T: C \rightarrow 2^{C} \backslash\{\emptyset\}$ be a set-valued mapping. Assume that the following conditions hold:

(i) $f(x, x) \geq 0$ for any $(x, x) \in C \times C$.

(ii) $T$ is upper $\succcurlyeq$-preserving and has upper bound $\succcurlyeq$-closed values. $x \nsucc C \backslash E$ for any $x \in E$.

(iii) $f(\cdot, y)$ is order-reversing for any $y \in C$ and $f(x, \cdot)$ is order-reversing for any $x \in C$.

$\{y \in T(x): f(x, y)<0\}$ is a complete $\succcurlyeq$-sublattice of $C$ for any $x \in C$.

(iv) There is $\hat{x} \in C \backslash E$ such that $\bigvee_{C} T(\hat{x}) \succcurlyeq \hat{x}$, or there is $\hat{x} \in E$ such that $\bigvee_{C} \Phi(\hat{x}) \succcurlyeq \hat{x}$.

Then QEP (1.1) is solvable.

Proof Define a set-valued mapping $\Phi: C \rightarrow 2^{C}$ by setting

$$
\Phi(x)=\{y \in T(x): f(x, y)<0\} .
$$

We only need to prove that $\Phi$ satisfies the assumption (iii) of Theorem 4.1. By the same argument as that in Theorem 3.2, $\Phi$ is upper $\succcurlyeq$-preserving. On the other hand, from the definition of $\Phi$, we have $\Phi(x) \subseteq T(x)$ for each $x \in C$. Since $\{y \in T(x): f(x, y)<0\}$ is a complete $\succcurlyeq$-sublattice of $C$ for any $x \in C$, we have $\bigvee_{C}\{y \in T(x): f(x, y)<0\} \in\{y \in T(x): f(x, y)<0\}$, that is, $\bigvee_{C} \Phi(x) \in \Phi(x)$, which implies that $\Phi$ has upper bound $\succcurlyeq$-closed values. Therefore, $\Phi$ satisfies the assumption (iii) of Theorem 4.1. From Theorem 4.1, there is a solution for quasi-equilibrium problem (1.1).

If $\bigvee_{C} C \in C$ and $T(x)=C$ for each $x \in C$, then we can deduce the following existence theorems for equilibrium problem (1.2) from Theorem 4.1 and Theorem 4.2.

Corollary 4.1 Let $(X, \succcurlyeq)$ be a poset and let $C$ be a chain-complete $\succcurlyeq$-sublattice of $X$ such that $\bigvee_{C} C \in C$. Let $f: C \times C \rightarrow R$ be a bifunction. Assume that the following conditions hold:

(i) $f(x, x) \geq 0$ for any $(x, x) \in C \times C$.

(ii) The set-valued mapping $\Phi: C \rightarrow 2^{C}$ defined by setting

$$
\Phi(x)=\{y \in C: f(x, y)<0\}
$$

is upper $\succcurlyeq$-preserving and has upper bound $\succcurlyeq$-closed values. 
(iii) There is $\hat{x} \in E$ such that $\bigvee_{C} \Phi(\hat{x}) \succcurlyeq \hat{x}$.

Then EP (1.2) is solvable.

Corollary 4.2 Let $(X, \succcurlyeq)$ be a poset and let $C$ be a chain-complete $\succcurlyeq$-sublattice of $X$ such that $\bigvee_{C} C \in C$. Let $f: C \times C \rightarrow R$ be a bifunction. Assume that the following conditions hold:

(i) $f(x, x) \geq 0$ for any $(x, x) \in C \times C$.

(ii) $f(\cdot, y)$ is order-reversing for any $y \in C$ and $f(x, \cdot)$ is order-reversing for any $x \in C$.

$\{y \in C: f(x, y)<0\}$ is a complete $\succcurlyeq$-sublattice of $C$ for any $x \in C$.

(iii) There is $\hat{x} \in C$ such that $\bigvee_{C}\{y \in C: f(\hat{x}, y)<0\} \succcurlyeq \hat{x}$.

Then EP (1.2) is solvable.

Remark 4.1 In Theorem 4.1, Theorem 4.2, Corollary 4.1 and Corollary 4.2 above, we always assume that $T$ is upper $\succcurlyeq$-preserving and $f(\cdot, y)$ is order-reversing for each $y \in C$ and $f(x, \cdot)$ is order-reversing for each $x \in C$. In fact, all of these conditions are used to guarantee that $\Psi$ is upper $\succcurlyeq$-preserving, so that we can obtain an isotone selection by Lemma 2.1. However, it is worthy to mention that these conditions related to upper $\succcurlyeq$-preservation are sufficient. That is, for getting an isotone selection, the set-valued mapping need not be upper order $\succcurlyeq$-preserving or lower $\succcurlyeq$-preserving. To see this, take $X=(R, \geq)$ and $C=[0,6]$. Define a set-valued mapping $\Gamma:[0,6] \rightarrow 2^{[0,6]}$ by setting

$$
\Gamma(x)=\{y \in R: \sin (x)+4 \geq y \geq \sin (x)+1\} .
$$

It is easy to check that $\Gamma$ is neither upper $\geq$-preserving nor lower $\geq$-preserving; however, we can find an isotone selection $F$ for $\Gamma$, where $F$ is defined by setting

$$
F(x)=\frac{1}{6} x+2
$$

Remark 4.2 We can also consider the case when $T$ is lower $\succcurlyeq$-preserving and $f(x, \cdot)$ is order-preserving for each $x \in C$ and $f(\cdot, y)$ is order-preserving for each $y \in C$. Applying Lemma 2.2, we can explore some existence theorems similar to Theorem 4.1 etc.

Now we consider the quasi-equilibrium problems on chain-complete posets. To this end, we need some order-theoretic fixed point theorems on chain-complete posets. The following result is usually called Abian-Brown fixed point theorem, which extends Lemma 4.1 from chain-complete lattices to chain-complete posets.

Lemma 4.2 (see [25]) Let $(X, \succcurlyeq)$ be a chain-complete poset, and let $F: X \rightarrow X$ be an orderpreserving single-valued mapping. If there is $\bar{x} \in X$ with $F(\bar{x}) \succcurlyeq \bar{x}$, then $F$ has a fixed point.

By using Lemma 4.2 and the methodology given in Theorem 3.1, we can prove the following result, which extends Theorem 4.2 from chain-complete lattices to chain-complete posets.

Theorem 4.3 Let $(X, \succcurlyeq)$ be a poset and let $C$ be a chain-complete subset of $X$. Let $f: C \times$ $C \rightarrow R$ be a bifunction and $T: C \rightarrow 2^{C} \backslash\{\emptyset\}$ be a set-valued mapping. Assume that the following conditions hold: 
(i) $f(x, x) \geq 0$ for any $(x, x) \in C \times C$.

(ii) $T$ is upper $\succcurlyeq$-preserving and has upper bound $\succcurlyeq$-closed values. $x \nsucc C \backslash E$ for any $x \in E$.

(iii) $f(\cdot, y)$ is order-reversing for any $y \in C$ and $f(x, \cdot)$ is order-reversing for any $x \in C$. $\{y \in T(x): f(x, y)<0\}$ is a complete $\succcurlyeq$-sublattice of $C$ for any $x \in C$.

(iv) There is $\hat{x} \in C \backslash E$ such that $\bigvee_{C} T(\hat{x}) \succcurlyeq \hat{x}$, or there is $\hat{x} \in E$ such that $\bigvee_{C}\{y \in T(\hat{x}): f(\hat{x}, y)<0\} \succcurlyeq \hat{x}$.

Then QEP (1.1) is solvable.

Remark 4.3 In a similar way, Theorem 4.1, Corollary 4.1 and Corollary 4.2 can be extended to chain-complete posets by using Lemma 4.2.

Very recently, Li [29] proved several extensions of Lemma 4.2 from single-valued mappings to set-valued mappings on chain-complete posets.

Lemma 4.3 (see [29]) Let $(X, \succcurlyeq)$ be a chain-complete poset and $F: X \rightarrow 2^{X} \backslash\{\emptyset\}$ be a set-valued mapping. Assume that:

A1. $\quad F$ is upper order-preserving.

A2. There is $y$ in $X$ with $u \succcurlyeq y$ for some $u \in F(y)$.

In addition, one of the following assumptions holds:

A3. $S F=\{z \in X: u \succcurlyeq z$ for some $u \in F(x)\}$ is an inductive poset for each $x \in X$.

$\mathrm{A}^{\prime} . \quad(F(x), \succcurlyeq)$ is inductive with a finite number of maximal elements for every $x \in X$.

A3". $F(x)$ has a maximum element for every $x \in X$.

A3'".$F(x)$ is a chain-complete lattice for each $x \in X$.

Then $F$ has a fixed point.

By using conditions A1, A2 and A3 in Lemma 4.3 and the methodology given in Theorem 3.2, we can obtain an existence theorem of solutions to quasi-equilibrium problems on chain-complete posets, where the isotone selection is dropped.

Theorem 4.4 Let $(X, \succcurlyeq)$ be a poset and let $C$ be a chain-complete subset of X. Let $f: C \times$ $C \rightarrow R$ be a bifunction and $T: C \rightarrow 2^{C} \backslash\{\varnothing\}$ be a set-valued mapping. Assume that the following conditions hold:

(i) $f(x, x) \geq 0$ for any $(x, x) \in C \times C$.

(ii) $T$ is upper $\succcurlyeq$-preserving and $T(x)$ has a maximum element for every $x \in C$. $x \nsucc C \backslash E$ for any $x \in E$.

(iii) $f(\cdot, y)$ is order-reversing for any $y \in C$ and $f(x, \cdot)$ is order-reversing for any $x \in C$, and $f\left(x, \bigvee_{C} T(x)\right)<0$ for each $x \in E$.

(iv) There is $y \in C \backslash E$ such that $y \preccurlyeq u$ for some $u \in T(y)$, or there is $y \in E$ such that $y \preccurlyeq u$ for some $u \in T(y)$ and $f(y, u)<0$.

Then QEP (1.1) is solvable.

On the other hand, by using conditions A1, A2 and A3"' in Lemma 4.3 and the methodology given in Theorem 3.2, we can establish another existence theorem of solutions to quasi-equilibrium problems on chain-complete posets. 
Theorem 4.5 Let $(X, \succcurlyeq)$ be a poset and let $C$ be a chain-complete subset of $X$. Let $f: C \times$ $C \rightarrow R$ be a bifunction and $T: C \rightarrow 2^{C} \backslash\{\emptyset\}$ be a set-valued mapping. Assume that the following conditions hold:

(i) $f(x, x) \geq 0$ for any $(x, x) \in C \times C$.

(ii) $T$ is upper $\succcurlyeq$-preserving and $T(x)$ is a chain-complete sublattice of $C$ for every $x \in C . x \nsucc C \backslash E$ for any $x \in E$.

(iii) $f(\cdot, y)$ is order-reversing for any $y \in C$ and $f(x, \cdot)$ is order-reversing for any $x \in C$.

(iv) There is $y \in C \backslash E$ such that $y \preccurlyeq u$ for some $u \in T(y)$, or there is $y \in E$ such that $y \preccurlyeq u$ for some $u \in T(y)$ and $f(y, u)<0$.

Then QEP (1.1) is solvable.

Remark 4.4 Based on Theorem 4.4 and Theorem 4.5, it is easy to deduce some corollaries for EP (1.2).

Remark 4.5 In the same manner, we can also consider the case when $T$ is lower $\succcurlyeq$-preserving and $f(x, \cdot)$ is order-preserving for each $x \in C$ and $f(\cdot, y)$ is order-preserving for each $y \in C$.

\section{Competing interests}

The authors declare that they have no competing interests.

\section{Authors' contributions}

All authors contributed equally to the writing of this paper. All authors read and approved the final manuscript.

\section{Author details}

${ }^{1}$ School of Applied Mathematics, Nanjing University of Finance and Economics, Nanjing, 210023, P.R. China. ${ }^{2}$ School of Mathematical Sciences, Anhui University, Hefei, 230601, P.R. China.

\section{Acknowledgements}

The authors sincerely thank the anonymous reviewers and editor for their valuable suggestions, which improved the presentation of this paper. The first author was supported financially by the National Natural Science Foundation of China (11071109). This work was also supported by the National Natural Science Foundation of China (11401296) and Jiangsu Provincial Natural Science Foundation of China (BK20141008).

Received: 28 December 2014 Accepted: 2 April 2015 Published online: 22 April 2015

\section{References}

1. Noor, MA, Oettli, W: On general nonlinear complementarity problems and quasi-equilibria. Matematiche 49, 313-331 (1994)

2. Blum, E, Oettli, W: From optimization and variational inequalities to equilibrium problems. Math. Stud. 63, 123-145 (1994)

3. Harker, PT: Generalized Nash games and quasi-variational inequalities. Eur. J. Oper. Res. 54, 81-94 (1991)

4. Harker, PT: A note on the existence of traffic equilibria. Appl. Math. Comput. 18, 277-283 (1986)

5. Lin, L, Park, S: On some generalized quasi-equilibrium problems. J. Math. Anal. Appl. 224, 167-181 (1998)

6. Park, S: Fixed points and quasi-equilibrium problems. Math. Comput. Model. 32, 1297-1304 (2000)

7. Fu, JY: Symmetric vector quasi-equilibrium problems. J. Math. Anal. Appl. 285, 708-713 (2003)

8. Khaliq, A: Implicit vector quasi-equilibrium problems with applications to variational inequalities. Nonlinear Anal. 63, 1823-1831 (2005)

9. Ding, XP: Quasi-equilibrium problems with applications to infinite optimization and constrained games in general topological spaces. Appl. Math. Lett. 13, 21-26 (2000)

10. Cubiotti, P: Existence of solutions for lower semicontinuous quasi-equilibrium problems. Comput. Math. Appl. 30 11-22 (1995)

11. Noor, MA: Auxiliary principle for generalized mixed variational-like inequalities. J. Math. Anal. Appl. 215, 75-85 (1997)

12. Lin, LJ, Huang, YJ: Generalized vector quasi-equilibrium problems with applications to common fixed point theorems and optimization problems. Nonlinear Anal. 66, 1275-1289 (2007)

13. Bianchia, M, Kassayb, G, Pinic, R: Ekeland's principle for vector equilibrium problems. Nonlinear Anal. 66, 1454-1464 (2007)

14. Ding, XP, Ding, TM: KKM type theorems and generalized vector equilibrium problems in noncompact FC-spaces. J. Math. Anal. Appl. 331, 1230-1245 (2007)

15. Al-Homidan, S, Ansari, QH: Fixed point theorems on product topological semilattice spaces, generalized abstract economies and systems of generalized vector quasi-equilibrium problems. Taiwan. J. Math. 15(1), $307-330$ (2011) 
16. Al-Homidan, S, Ansari, QH, Yao, J-C: Collectively fixed point and maximal element theorems in topological semilattice spaces. Appl. Anal. 96(6), 865-888 (2011)

17. Lin, L-J, Yu, Z-T, Ansari, QH, Lai, L-P: Fixed point and maximal element theorems with applications to abstract economies and minimax inequalities. J. Math. Anal. Appl. 284, 656-671 (2003)

18. Lin, L-J, Ansari, QH: Collective fixed points and maximal elements with applications to abstract economies. J. Math. Anal. Appl. 296, 455-472 (2004)

19. Fujimoto, T: An extension of Tarski's fixed point theorem and its application to isotone complementarity problems. Math. Program. 28, 116-118 (1984)

20. Chitra, A, Subrahmanyam, P: Remarks on nonlinear complementarity problem. J. Optim. Theory Appl. 53, 297-302 (1987)

21. Borwein, J, Dempster, M: The linear order complementarity problem. Math. Oper. Res. 14, 534-558 (1989)

22. Nishimura, H, Ok, EA: Solvability of variational inequalities on Hilbert lattices. Math. Oper. Res. 37(4), 608-625 (2012)

23. Li, J, Ok, EA: Optimal solutions to variational inequalities on Banach lattices. J. Math. Anal. Appl. 388, 1157-1165 (2012)

24. Li, J, Yao, JC: The existence of maximum and minimum solutions to general variational inequalities in Hilbert lattices. Fixed Point Theory Appl. (2011). doi:10.1155/2011/904320

25. Ok, EA: Order theory (2011). https://files.nyu.edu/eo1/public/books.html

26. Meyer-Nieberg, P: Banach Lattices. Universitext. Springer, Berlin (1991)

27. Smithson, RE: Fixed points of order preserving multifunctions. Proc. Am. Math. Soc. 28, 304-310 (1971)

28. Tarski, A: The lattice theoretical fixed point theorem and its applications. Pac. J. Math. 5, 285-309 (1955)

29. Li, J: Several extensions of the Abian-Brown fixed point theorem and their applications to extended and generalized Nash equilibria on chain-complete posets. J. Math. Anal. Appl. 409, 1084-1092 (2014)

\section{Submit your manuscript to a SpringerOpen ${ }^{\circ}$ journal and benefit from:}

- Convenient online submission

- Rigorous peer review

- Immediate publication on acceptance

- Open access: articles freely available online

- High visibility within the field

- Retaining the copyright to your article 